\title{
Perspectives on Breast Cancer Management: Tailoring Treatment to the Individual Patient
}

\author{
Christoph Thomssen \\ Klinik und Poliklinik für Gynäkologie, Martin-Luther-Universität Halle/Saale, Germany
}

\section{Defining Risk of Recurrence in the Individual Patient}

In everyday clinical practice, the decision to treat a breast cancer patient depends on the estimation of her individual risk of relapse. In an optimal setting, the patient at risk can be identified and treated with agents specifically targeted to her individual cancer profile. In reality, however, only a very small group of patients can be sufficiently characterized on the basis of clinical features such as tumor size, nodal status, grading, or age to have a minimal risk of relapse so that they can be spared from adjuvant systemic treatment and side effects associated with therapy. The vast majority of patients is recognized as being at a certain risk and, therefore, treated maximally. Current international treatment guidelines recommend adjuvant systemic treatment for a large fraction of breast cancer patients, including all patients with lymph node-positive disease and up to $90 \%$ of patients with lymph node-negative disease [1-4].

Complex individual risk prediction models which combine clinical and histopathological features have been constructed to predict probability of relapse and treatment response. Moreover, two Dutch study groups developed gene expression profiles of breast cancers each comprising about 70 genes to separate breast cancer patients into subgroups with good versus poor prognosis gene signature [4-7]. Unfortunately, these two prognostic expression signatures have very little overlap and contain only two genes in common. They, therefore, appear to be of little clinical relevance to date and not yet ready for routine application in treatment decisions [8]. The Amsterdam gene signature is currently validated in a prospective and randomized fashion versus the common clinical-pathological criteria in the European study MINDACT [9].

An alternative approach is evaluated in the ongoing study NNBC 3-Europe which aims at validating the urokinase-type plasminogen activator (UPA) and its inhibitor PAI-1 for clinical routine risk assessment and tailoring adjuvant chemotherapy in node-negative high-risk patients [10]. uPA and PAI are components of the plasminogen activator system that plays a role in cell invasion and angiogenesis in a variety of solid tumors. Increased levels of uPA and/or PAI-1 could be correlated with poor clinical outcome [11, 12]. Finally, the molecular diagnostic tool Oncotype DX (Genomic Health Inc., Redwood City, CA, USA) that quantifies the likelihood of recurrence in patients with newly diagnosed early-stage breast cancer and assesses the benefit from chemotherapy by analyzing a panel of 21 genes is validated in the TAILORx trial which will recruit over 10,000 patients in over 1,000 US centers. Meanwhile, the invasion markers uPA/PAI-1 and the multigene assay Oncotype DX have been accepted and are recommended as prognostic tools in breast cancer patients by the recently updated ASCO guidelines on tumor markers [13]

\section{Pharmacogenetics to Predict Treatment Response}

Possible future markers for the prediction of the efficacy of a given therapy are gene polymorphisms, e.g. in the metabolizing cytochrome peroxidase P450 (CYP) system. The importance of the CYP system for the response to tamoxifen has recently been elucidated: The prodrug tamoxifen is metabolized to the active metabolite endoxifen by CYP2D6 (fig. 1). Patients with CYP2D6 polymorphisms resulting in a low activity of this enzyme (slow metabolizers) have a significantly shorter relapse-free survival with adjuvant tamoxifen therapy than patients with wild-type CYP2D6 and normal enzyme activity resulting in higher plasma concentrations of the active metabolite (extensive metabolizers) [14, 15]. Based on these findings, in the future, CYP2D6 genotyping may identify patients who are less likely to benefit from adjuvant tamoxifen therapy and for whom alternative agents are advisable.

The different pattern of tamoxifen metabolization might be reflected by the incidence of hot flashes which is probably higher in patients without CYP2D6 polymorphism and higher plasma levels of endoxifen. Indeed, the frequency of hot flash-

\begin{tabular}{ll}
\hline KARGER & $\oplus$ 2008 S. Karger GmbH, Freiburg \\
Fax +497614520714 & Accessible online at: \\
$\begin{array}{l}\text { E-mail Information@Karger.de } \\
\text { www.karger.com }\end{array}$ & www.karger.com/brc \\
&
\end{tabular}




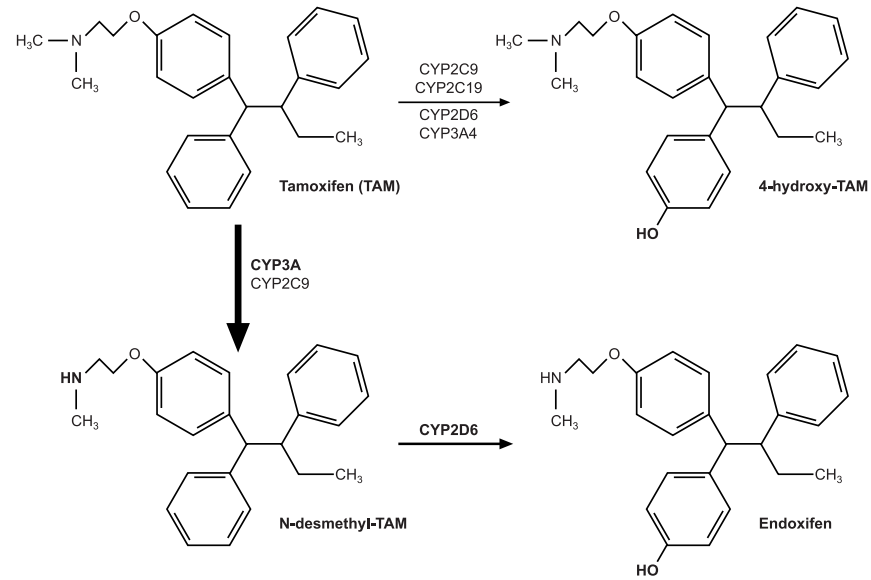

Fig. 1. Tamoxifen metabolism by CYP2D6 (adapted from [14]).

es was recently identified as a predictive marker of outcome by Mortimer et al. [16]: In their study with nearly 900 patients and a follow-up of more than 7 years, the recurrence-free survival was stronger related to the incidence of hot flashes than to age, hormone receptor status, and tumor stage.

\section{Tailoring Treatment to Cancer Targets}

In the last couple of years, advances in the molecular understanding of tumor growth and tumor neoangiogenesis have resulted in the identification of rational targets for cancer therapy. An attractive approach is the interference with the sprouting of new vasculature, as already small tumors exceeding a volume of $0.5 \mathrm{~cm}^{3}$ are dependent on new vessel formation for a sufficient supply with oxygen and nutrients. Once the cancer cell has developed a proangiogenic phenotype (angiogenic switch) induced by hypoxia in the growing tumor, proangiogenic mediators are expressed and secreted from the tumor, vascular endothelial growth factor (VEGF) being one of the most prominent ones. VEGF has emerged as an important prognostic marker in node-negative breast cancer [17]: Higher levels of this growth factor correlate significantly with shorter recurrence-free and overall survival $(\mathrm{p}=0.0113$ and $\mathrm{p}=$ 0.0075 , respectively).

The inhibition of tumor angiogenesis by blocking the mediators or their receptors involved in new vessel formation has evolved as a promising new treatment approach. Until now, several strategies have been successfully used in the clinical setting. The monoclonal antibody bevacizumab works by binding VEGF in the circulation, thus preventing binding of this ligand to its receptor VEGF-R on the surface of endothelial cells and activation of downstream-signaling pathways. In metastatic breast cancer the combination of bevacizumab with paclitaxel resulted in a significant prolongation of progressionfree survival compared to paclitaxel alone $(p<0.001)[18]$. Bevacizumab is currently tested in the adjuvant setting in patients with triple-negative breast cancer known to have a very poor prognosis. In the BEATRICE trial, patients are randomized to bevacizumab or placebo after completion of standard adjuvant chemotherapy. Nevertheless, one has to keep in mind that, in contrast to anti-ErbB2 (anti-HER2) therapy, there is still no molecular factor predicting response to bevacizumab. Therefore, in the BEATRICE trial, the targeted agent bevacizumab is used unselectively in poor-risk patients. Hence, this strategy is inconsistent with current efforts of tailoring therapy to carefully selected patients based on biological or clinical factors. Moreover, in the adjuvant setting, treatment is directed against dormant tumor cells. Whether an anti-angiogenic treatment is a successful approach for dormant tumor cells is still unknown.

In contrast to monoclonal antibodies, tyrosine kinase inhibitors such as lapatinib or pazopanib are small molecules able to penetrate the cell membrane and to competitively inhibit the intracellular tyrosine kinase domain of two (lapatinib) or multiple (pazopanib) different growth factor receptors. As intracellular signaling pathways are redundant, blocking of only one of these pathways has turned out to be insufficient for completely controlling tumor cell proliferation. On the basis of in vitro data, combination therapies with several molecular drugs directed against different targets of the tumor cells appear to be a more promising approach. The clinical relevance of such an approach is currently evaluated in the study VEG 108838: Women with ErbB2-overexpressing inflammatory breast cancer who have failed prior chemotherapy with or without trastuzumab will be randomized to the tyrosine kinase inhibitor lapatinib as monotherapy or in combination with pazopanib.

In the adjuvant setting, the 4-arm ALTTO trial just started to compare lapatinib and trastuzumab as monotherapies, or in combination, or in sequence. Results of these trials will be available in approximately 5 years and might change treatment attitudes in the adjuvant therapy improving the prognosis for women with ErbB2-overexpressing breast tumors.

Ongoing studies in the neoadjuvant situation will be the first to present efficacy data on different types of combination treatments with chemotherapy and targeted therapy. One example is the German GEPARQUINTO trial which evaluates standard chemotherapy in combination with different antiErbB2 therapies (namely lapatinib and trastuzumab), an mTOR inhibitor (everolimus), and the antiangiogenic drug bevacizumab.

In conclusion, a more precise tailoring of adjuvant therapies to the individual patient, based on individual risk prediction, is urgently needed. At the moment, only immunohistochemistry and expression assays of single well-defined genes as well as a pharmacogenetic characterization of the patient appear to be of clinical relevance in the routine practice. The development of additional drugs targeting ErbB2, a panel of different growth factors, and their receptors will enable us to use combined treatment approaches hopefully resulting in a more potent inhibition of tumor cell growth and invasion. 


\section{References}

1 Early Breast Cancer Trialists' Collaborative Group Polychemotherapy for early breast cancer: an overview of the randomized trials. Lancet 1998;352: 930-942.

2 Early Breast Cancer Trialists' Collaborative Group: Tamoxifen for early breast cancer: an overview of the randomized trials. Lancet 1998;351:1451-1467.

3 Goldhirsch A, Wood WC, Gelber RD, et al. Progress and promise: highlights of the international expert consensus on the primary therapy of early breast cancer 2007. Ann Oncol 2007;18:1133-1144.

4 Eifel P, Axelsson JA, Costa J, et al.: National Institutes of Health Consensus Development Conference Statement: adjuvant therapy for breast cancer November 1-3 2000. J Natl Cancer Inst 2001;93: 979-989.

5 Wang Y, Klijn JG, Zhang Y, et al.: Gene-expression profiles to predict distant metastasis of lymphnode-negative primary breast cancer. Lancet 2005 ; 671-679.

6 Van't Veer LJ, Dai H, van de Vijver MJ, et al.: Gene expression profiling predicts clinical outcome of breast cancer. Nature 2002;415:530-536.

7 Van de Vijver MJ, He YD, van't Veer EJ, et al.: A gene-expression signature as a predictor of survival in breast cancer. N Engl J Med 2002;347:1999-2009.
Ein-Dor L, Kela I, Getz G, et al.: Outcome signature genes in breast cancer. Is there a unique set? Bioinformatics 2005;21:171-178.

9 Piccart MJ, Loi S, van't Veer L, et al.: Multi-center external validation study of the Amsterdam 70gene prognostic signature in node negative untreated breast cancer: are the results still outperforming clinical-pathological criteria? Breast Cancer Res Treat 2004;88(suppl 1):abstr 38.

10 Vetter M, Kantelhardt EJ, Annecke K, et al.: Invasionsfaktoren uPA/PAI-1 im Tumorgewebe bei Patientinnen mit primärem Mammakarzinom: Von Forschungsergebnissen zur klinischen Anwendung am Beispiel der NNBC 3-Europe Studie. GeburtshFrauenheilk 2007;67:1144-1152.

11 Jänicke F, Schmitt M, Ulm K, et al.: Urokinase-type plasminogen activator antigen and early relapse in breast cancer. Lancet 1989;2:1049.

12 Duffy MJ, Reilly D, O'Sullivan, et al.: Urokinaseplaminogen activator: a new and independent prognostic marker in breast cancer. Cancer Res 1990;50: 6827-6829.

13 Harris L, Fritsche H, Mennel R, et al.: American Society of Clinical Oncology 2007 update of recommendations for the use of tumor markers in breast cancer. J Clin Oncol 2007;25:5268-5312.
4 Stearns V, Johnson MD, Rae JM, et al.: Active tamoxifen metabolite plasma concentrations after coadministration of tamoxifen and the selective serotonin reuptake inhibitor paroxetine. J Natl Cancer Inst 2003;95:1758-1764.

15 Goetz MP, Rae JM, Suman VJ, et al.: Pharmacogenetics of tamoxifen biotransformation is associated with clinical outcome of efficacy and hot flashes. J Clin Oncol 2005;23:9312-9318.

16 Mortimer J, Flatt S, Parker B, et al.: Tamoxifen, hot flashes and recurrence in breast cancer: support for pharmacogenetics. Proc Am Soc Clin Oncol 2007; 25(18S):abstr 500.

17 Linderholm B, Tavelin B, Grankvist K, et al.: Does vascular enothelial growth factor (VEGF) predict local relapse and survival in radiotherapy-treated node-negative breast cancer? Br J Cancer 1999;81: 727-733.

18 Miller KD, Wang M, Gralow J, et al.: A randomized phase III trial of paclitaxel versus paclitaxel plus bevacizumab as first-line therapy of locally recurrent or metastatic breast cancer: a trial coordinated by the Eastern Cooperative Oncology Group (E2100). SABCS, San Antonio, TX, 2005, abstr 3. 